\title{
Prevalence of carrier state theileriosis in lactating cows
}

\author{
Niranjana Sahoo사 ${ }^{1}$ Bikash Kumar Behera², Hemant Kumar Khuntia ${ }^{3}$ and Manojita Dash²
}

1. Department of Epidemiology \& Preventive Medicine, College of Veterinary Science \& Animal Husbandry, Orissa University of Agriculture and Technology, Bhubaneswar, 751003, Odisha, India; 2. Centre for Wildlife Health, College of Veterinary Science \& Animal Husbandry, Orissa University of Agriculture and Technology, Bhubaneswar, 751003, Odisha, India; 3. ICMR-Regional Medical Research Centre, Department of Health Research, Ministry of Health \& Family Welfare, Government of India, Bhubaneswar, 751023, Odisha, India.

Corresponding author: Niranjana Sahoo, e-mail: niranjanasahoo@hotmail.com

Co-authors: BKB: bikash2249@gmail.com,HKK: khuntia_hk@yahoo.co.in, MD: manojita.dash@gmail.com Received: 20-07-2017, Accepted: 02-11-2017, Published online: 14-12-2017

doi: 10.14202/vetworld.2017.1471-1474 How to cite this article: Sahoo N, Behera BK, Khuntia HK, Dash M (2017) Prevalence of carrier status theileriosis in lactating cows, Veterinary World, 10(12): 1471-1474.

\begin{abstract}
Aim: The objective of this study was to examine the carrier status of theileriosis among apparently healthy cross-bred jersey cattle population of Odisha using conventional blood smear examination and polymerase chain reaction (PCR).

Materials and Methods: A total of 34 apparently healthy cross-bred Jersey lactating cows were considered in this study. Blood samples were subjected to microscopic examination after staining with Giemsa stain and PCR based molecular diagnosis using two sets of primer, i.e., N516/N517 and TorF1/TorF2 specific for Theileria annulata and Theileria orientalis, respectively.
\end{abstract}

Results: Examination of blood samples revealed presence of theileria parasites to a magnitude of $20.59 \%$ for $T$. annulata, $8.82 \%$ for $T$. orientalis, and $2.94 \%$ for both.

Conclusion: Molecular diagnosis was found to be much more sensitive than conventional method for diagnosis of theileriosis. T. annulata was found to be the predominant species affecting the exotic cattle. T. orientalis was detected in apparently healthy cows.

Keywords: bovine theileriosis, carrier state, polymerase chain reaction, Theileria annulata, Theileria orientalis.

\section{Introduction}

Hemoprotozoan diseases in general and theileriosis in particular are considered as a serious economic threat for dairy farmers due to its adverse effects in the form of mortality and low productivity particularly in the tropical and subtropical regions of the world [1].

Continuous change in climatic conditions in the past few decades resulting in high environmental temperature and humidity has favored tick multiplication and thereby a gradual surge in the incidence of tick-borne menace [2]. Increased population and introduction of the exotic/crossbred cattle population, especially in the endemic areas, has magnified susceptibility to theileriosis. Predisposing factors linked with stress such as high production, low nutrition, poor housing, unhygienic indoor condition, and development of drug resistance to acaricidal agents have further intensified the adverse impact of theileriosis.

India has long been established as a victim of theileriosis. A review of the literature on the incidence

\footnotetext{
Copyright: Sahoo, et al. Open Access. This article is distributed under the terms of the Creative Commons Attribution 4.0 International License (http://creativecommons.org/licenses/by/4.0/), which permits unrestricted use, distribution, and reproduction in any medium, provided you give appropriate credit to the original author(s) and the source, provide a link to the Creative Commons license, and indicate if changes were made. The Creative Commons Public Domain Dedication waiver (http://creativecommons.org/ publicdomain/zero/1.0/) applies to the data made available in this article, unless otherwise stated.
}

of theileriosis in India over the past four decades has revealed its existence in most states of India [3], including Odisha [4,5]. Most of the research work in India are focused on bovine tropical theileriosis caused by Theileria annulata. However, during the past few years, reports on Theileria orientalis associated clinical infections have been accumulated from different states of India such as Tamil Nadu, Kerala, and Assam [6-9]. Thus, identification of species-specific theileriosis is essentially required to unveil the picture of bovine theileriosis in the areas of interest. Besides, differential diagnosis of the diseases having clinical findings similar to each other is of utmost importance to avoid polypharmacy especially in high yielders having enhanced vulnerability to theileriosis [10].

Clinical signs such as fever, inappetence, anemia, coughing, dyspnea, reduced milk yield, swollen subcutaneous lymph nodes, abortion, icterus, hemoglobinuria and soil licking, raise suspicion of clinical theileriosis, and pave way for confirmatory diagnosis whereas the animals with low infectivity remain undiagnosed and continue to be a constant source of infection for other susceptible hosts. Keeping the above facts in forefront, the present study was conducted to diagnose occult or carrier status, if any, by microscopy and polymerase chain reaction among crossbred lactating cows in an area of Odisha, India, reported to be endemic for theileriosis. 


\section{Materials and Methods}

\section{Ethical approval}

Laboratory tests required for the investigation were in accordance to the guidelines provided in the Institutional Animal Ethics Committee, and were performed in the Department of Veterinary Epidemiology and Preventive Medicine. The study period extended from March 2016 to February 2017.

\section{Experimental design}

Blood samples were collected from apparently healthy crossbred Jersey lactating cows reared in small private farms (head size: 2-8) of Niali block of Cuttack district of Odisha bearing GPS coordinates $20.1411^{\circ} \mathrm{N}$ and $86.0606^{\circ} \mathrm{E}$. Cows selected in the study were between $3^{\text {rd }}$ and $6^{\text {th }}$ lactation, within 2 months of parturition and with yielding record of minimum $10.0 \mathrm{~L}$ per day. A total of 34 cows were sampled in the study. $1 \mathrm{~mL}$ blood sample from each cow was collected properly from jugular vein in EDTA coated vacutainer tubes during morning hours between 5.00 and $7.00 \mathrm{am}$. Thin blood smears were prepared from the freshly collected blood samples, and the rest was stored at $4{ }^{\circ} \mathrm{C}$ for subsequent use in PCR.

\section{Microscopic examination}

Thin blood smear was prepared taking one drop $(50 \mu 1)$ of blood on a clean glass slide and spreading it with another spreader slide at an angle of $45^{\circ}$. Prepared smear was fixed with methanol for $5 \mathrm{~min}$ followed by flooding with $10 \%$ Giemsa's solution for $40 \mathrm{~min}$. Blood smears were carefully examined for Theileria parasites under the oil immersion lens $(100 \times$ magnifications). Presence of piroplasm(s) inside the erythrocytes was considered positive for Theileria spp. infection.

\section{DNA extraction and PCR}

Genomic DNA was extracted from each blood sample using commercially available DNA mini kit (QIAGEN, USA). According to the manufacturer's instructions, $200 \mu \mathrm{l}$ of whole blood was used for each sample. The concentration of extracted DNA was checked by agarose gel electrophoresis. This purified DNA was used as a template for the PCR. Two sets of primers were used in the investigation, i.e., N516/ N517 (primer set A) for T. annulata and TorF1/TorF2 (primer set B) for T. orientalis (Table-1). PCR was performed in a final reaction volume of $25 \mu 1$ reaction mixtures containing $2 \mu \mathrm{l}$ DNA sample (using $30 \mathrm{ng} / \mu \mathrm{l}$ template in case of DNA reference samples), $50 \mathrm{mM}$ $\mathrm{KCl}, 10 \mathrm{mM}$ Tris- $\mathrm{HCl}(\mathrm{pH} 8.3), 1.5 \mathrm{mM} \mathrm{MgCl}_{2}$, $200 \mu \mathrm{M}$ of dNTP mix, $20 \mathrm{pmol}$ of each primer, and
$0.5 \mathrm{U}$ Taq polymerase enzyme and sterile distilled water up to $25 \mu 1$. The reaction mixture was placed on a heating block of a programmable thermocycler (Life technologies, Thermo Fischer scientific). After a denaturation step of $5 \mathrm{~min}$ at $94^{\circ} \mathrm{C}$, each of 30 cycle consisted of $1 \mathrm{~min}$ at $94^{\circ} \mathrm{C}, 1 \mathrm{~min}$ at $55^{\circ} \mathrm{C}, 1 \mathrm{~min}$ at $72^{\circ} \mathrm{C}$ followed by $10 \mathrm{~min}$ at $72^{\circ} \mathrm{C}$ for $T$. annulata whereas for $T$. orientalis after denaturation of $5 \mathrm{~min}$ at $94^{\circ} \mathrm{C}$, each of the 35 cycles consisted of $1 \mathrm{~min}$ at $94^{\circ} \mathrm{C}, 30 \mathrm{~s}$ at $55^{\circ} \mathrm{C}, 45 \mathrm{~s}$ at $72^{\circ} \mathrm{C}$, and final extension of $5 \mathrm{~min}$ at $72^{\circ} \mathrm{C}$. Positive control and negative control samples were run along with the test samples. The amplification products were subjected to electrophoresis on $1.5 \%$ agarose gel with a ladder and the amplified products were visualized using gel documentation system.

Amplified products were randomly selected and sent to Bhat bio-tech India private limited, Bangalore, Karnataka, for sequencing.

\section{Results}

Morphologically, under microscopic examination of blood smears positive for Theileria spp. infections, intraerythrocytic bodies appeared in the form of dot shape in $T$. annulata and slightly elongate in case of T. orientalis [11]. Examination of 34 Giemsa stained blood smears from apparently healthy cows, $3(8.82 \%)$ samples showed the presence of Theileria spp. parasite. Schizonts could not be demonstrated in any of these blood smears.

Further examination of the blood samples on PCR showed a different picture. PCR reactions showing a product size of 721 bp by primers N516/N517 and a product size of $776 \mathrm{bp}$ by primers TorF $1 /$ TorR 1 were considered positive for T. annulata (Figure-1) and T. orientalis (Figure-2), respectively. Of all the 34 blood samples of the apparently healthy lactating cows, the presence of $T$. orientalis and $T$. annulata was found in $7(20.59 \%)$ and $3(8.82 \%)$ cows, respectively, while both the species were detected in a solitary case (2.94\%). These findings established the carrier stage of T. annulata and T. orientalis, either single and/or mixed form, in 11 (32.35\%) crossbred Jersey Milch cows.

Sequencing of the amplified PCR products confirmed that the product has 100\% sequence homology with $T$. annulata and T. orientalis.

\section{Discussion}

Odisha is situated along the eastern coast of India between $18^{\circ} 2^{\prime}$ and $22^{\circ} 6^{\prime} \mathrm{N}$ latitude and $82^{\circ} 8^{\prime}$ and $87^{\circ} 6^{\prime} \mathrm{E}$ longitude. The state experiences moderately

Table-1: Primer sets used for PCR.

\begin{tabular}{llccc}
\hline Oligo name & Sequence $\left(\mathbf{5}^{\prime} \rightarrow \mathbf{3}^{\prime}\right)$ & Product size & Target genome & References \\
\hline N516 & GTAACCTTAAAAACGT & $721 \mathrm{bp}$ & T. annulata specific & [21] \\
N517 & GTTACGAACATGGGTT & $776 \mathrm{bp}$ & T. orientalis specific & [22] \\
Tor F1 & CTTTGCCTAGGATACTTCCT & & & \\
Tor R1 & ACGGCAAGTGGTGAGAACT & & & \\
\hline
\end{tabular}

PCR=Polymerase chain reaction, $T$. annulata=Theileria annulata, $T$. orientalis=Theileria orientalis 


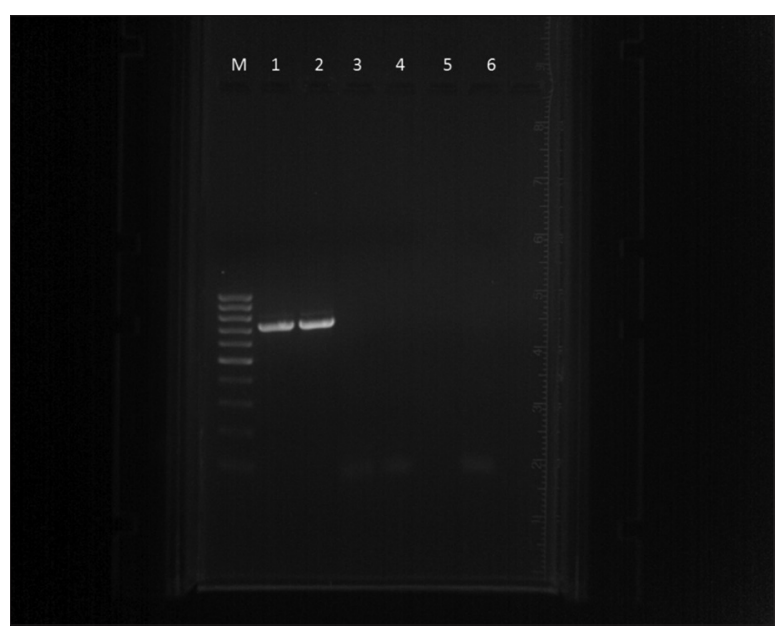

Figure-1: Agarose gel electrophoresis of polymerase chain reaction products: Lane $\mathrm{M}$ - 100 bp Marker, Lane 1 - positive control for Theileria annulata, Lane 2 - positive sample, Lane 3-5 - Negative samples, and Lane 6 Negative control.

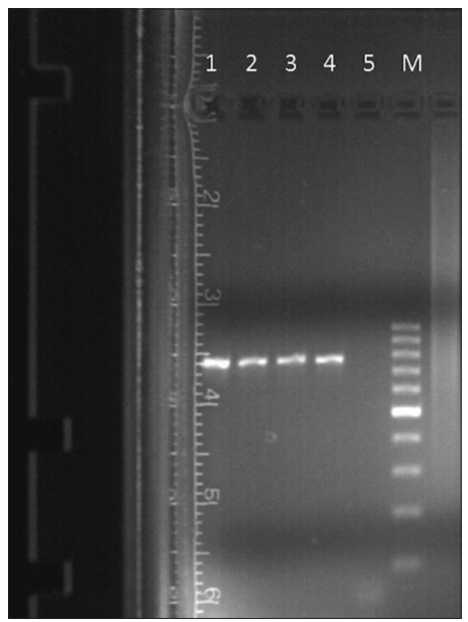

Figure-2: Agarose gel electrophoresis of polymerase chain reaction products: Lane 1 - positive control for Theileria orientalis, Lane 2-4 - positive samples, Lane 5 - Negative control and Lane M - Marker DNA (100 bp).

hot and very humid climate favoring rapid multiplication of vectors and thereby propagation of vector-borne diseases in the state. Of such diseases, bovine theileriosis is one of the most destructive obstacles to livestock production with massive economic repercussions [12]. At least five species of Theileria spp. (T. annulata, Theileria parva, Theileria taurotragi, Theileria velifera and members of Theileria sergenti/ orientalis/buffalo group) have been found to infect cattle. The present study unveiled the carrier status of T. annulata and T. orientalis in Odisha, a coastal state in eastern part of India.

Although the conventional Giemsa stained blood smear examination considered to be the gold standard method for identification of intraerythrocytic and Schizont stage of Theileria spp. parasite, this method is rarely successful in case of carrier animals [13]. This is in line with the findings in the present study where very low $(8.82 \%)$ proportion of positivity was recorded as against $32.35 \%$ in molecular tests. Moreover, by microscopic examination, it is difficult to discriminate different species of Theileria spp. that may occur either as single or mixed form within the same bovine host [14]. The serological tests are also not suitable due to cross-reactivity with other Theileria spp. [15] and inability to distinguish between active carriers and animals with antibodies due to prior infections [16]. Limited information is available regarding the role played by these carrier animals with respect to their ability to transmit the disease since they usually remain undetected being asymptomatic [17]. It is reported that most assays may detect $>400,000$ parasites/L blood and natural parasitaemia distribution in carrier state animals seems to be above this limit of detection, suggesting that most molecular assays should be able to detect the majority of infected individuals under endemic conditions. To overcome these constraints, PCR was preferred for its high sensitivity and the ability to amplify even a minute concentration of parasitic DNA in the blood enabling the detection of carrier animals even in endemic conditions [18].

Using PCR, T. orientalis was found to be the predominant species prevailing in the state of Odisha. Besides T. annulata, the prevalence of $T$. orientalis infections was seen for the first time in Odisha which has earlier been documented in Tamil Nadu [7] and Assam [11]. Mixed infection was found in one case which has previously been reported in Tamil Nadu [8].

Appearance of carrier animals in endemic areas is a matter of concern. Carrier state is usually chronic in nature [2]. Such category of infected animals with the tick feeding on them might be the source of infection when reared together with healthy cattle. Moreover, disease outbreak due to $T$. orientalis may represent a hidden burden to livestock productivity in regions where infection is endemic [19]. T. orientalis has also been found capable of being mechanically transmitted to healthy cattle by minute volumes of blood through intravenous inoculation as well as through biting arthropods [20]. Hence, it is not unwise to infer that this mode of transmission, not unlikely in field conditions, might be contributing a lot in dissemination of the disease through multiple use of unsterilized needles during the therapeutic process. The present PCR based assay helped to throw light on carrier stage of the Theileria spp. both T. annulata and T. orientalis in Odisha. The results of the study would be of immense help in proper planning and effective control of theileria infection.

\section{Conclusion}

Examination of blood samples from 34 apparently healthy cross-bred Jersey lactating cows in an endemic area exposed the presence of Theileria spp. in $3(8.82 \%)$ on blood staining and through PCR in $11(32.35 \%)$ cows, thereby confirmed its carrier status, 
either single or mixed form. T. orientalis constituted the major share as compared to T. annulata.

\section{Authors' Contributions}

MD collected the samples from the field and carried out the preliminary laboratory work. BKB performed the molecular work of the study under the guidance of HKK. NS supervised the entire laboratory activities. NS and HKK prepared manuscript whereas former revised the same. All authors read and approved the final manuscript.

\section{Acknowledgments}

The authors are thankful to the Dean, College of Veterinary Science and Animal Husbandry, as well as Dean of Research, Orissa University of Agriculture and Technology, Bhubaneswar, for providing necessary support to carry out the investigation with financial support under Rashtriya Krishi Vikas Yojana (Grant no. 198/DR dated 15-01-2014).

\section{Competing Interests}

The authors declare that they have no competing interests.

\section{References}

1. Inci, A., Ica, A., Yildirim, A., Vatansever, Z., Cakmak, A., Albasan, H., Cam, Y., Atasever, A., Sariozkan, S. and Duzlu, O. (2009) Economic impact of tropical theileriosis in the Cappadocia region of Turkey. Türkiye Parazitol. Derg., 33: 273-279.

2. Kohli, S., Atheya, UK. and Thapliyal, A. (2014) Prevalence of theileriosis in cross-bred cattle: Its detection through blood smear examination and polymerase chain reaction in Dehradun district, Uttarakhand, India. Vet. World, 7: $168-171$.

3. NDDB. (2015) Animal Health Updates, Q. Newsl., 3(4):1-4.

4. Panda, S.K., Sahu, B., Ranjan, R., Acharya, A.P. and Rath, S.K. (2012) Prevalence and clinicopathological study of theileriosis in bovine in coastal areas of Orissa. Indian $J$. Vet. Pathol., 35(2): 17-20.

5. Acharya, A.P., Panda, SK. and Prusty, BK. (2015) Molecular Diagnosis of Bovine Tropical Theileriosis in Cattle in Odisha. Presented at IAVP Conference, Gannavaram, Andhra Pradesh.

6. Aparna, M., Ravindran, R., Vimalkumar, MB., Laxmanan, B., Rameshkumar, P., Ajithkumar, KG., Promod, K., Ajithkumar, S., Ravishankar, C., Devada, K., Subramanian, H., George, J. and Ghosh, S. (2011) Molecular characterization of Theileria orientalis causing fatal infection in crossbred adult bovines of South India. Parasitol. Int., 60(4): 524-529.

7. Anupama, R., Srinivsasan, SR. and Parthiban, M. (2015). Molecular studies on theileriosis and identification of Theileria orientalis in India using PCR. Indian Vet. J., 92(2): 9-11.
8. Vinodkumar, K., Shyma, V., Justin, DK., Ashok, S., Anu, JP., Mini, K., Muhammedkutty, V., Sasidharan, S. and Chullipparambil, S. (2016) Fatal Theileria orientalis N2 genotype infection among Asian water buffaloes (Bubalus bubalis) in a commercial dairy farm in Kerala, India. Camb. Univ. Press Parasitol., 143(1): 69-74.

9. Kakati, P., Sarmah, P.C., Ray, D., Bhattacharjee, K., Sharma, R.K., Barkalita, L.M., Sarma, D.K., Baishya, B.C., Borah, P. and Stanley, B. (2015) Emergence of oriental theileriosis in cattle and its transmission through Rhipicephalus (Boophilus) microplus in Assam, India. Vet. World, 8(9): 1099-1104.

10. Kakati, P., Sarmah, P.C., Bhattacharjee, K., Bhuyan, D. and Baishya, B.C. (2015) Molecular detection and associated pathogenesis in a fatal case of Theileria orientalis infection in India: Probable circulation of a virulent strain and stress associated factors. Int. J. Rec. Sci. Res., 6: 4235-4239.

11. Soulsby, E.J.L. (1982) Heminths, Arthropods and Protozoa of Domesticated Animals. $7^{\text {th }}$ ed. UK Blackwell Scientific Publications, London.

12. Urrani, A.Z. (2008) Identification of Ticks and Detection of Blood Protozoa in Friesian Cattle by Polymerase Chain Reaction Test and Estimation of Blood Parameters in District Kasur, Pakistan. Springer Science and Business Media, Bouvet Island (B.V). p441-447.

13. Parthiban, M., Saranya, R., Magesh, M. and Raman, M. (2010) Detection of Theileria parasite in cattle of Tamilnadu using nested PCR. Tamilnadu J. Vet. Anim. Sci., 6: 162-165.

14. Anonymous. (2014) OIE Terrestrial Manual. Ch. 2.4.16.

15. Dolan, T.T. (1986) Chemotherapy of east coast fever: The long term weight changes, carrier state and disease manifestations of parvaquone treated cattle. J. Comp. Pathol., 96: 137.

16. Roy, K.C., Ray, D., Bansal, G.C. and Singh, R.K. (2000) Detection of Theileria annulata carrier cattle by PCR. Indian J. Exp. Biol., 28: 283-284.

17. Mans, B.J., Pienaar, R. and Latif, A.A. (2015) A review of Theileria diagnostics and epidemiology. Int. J. Parasitol. Parasites Wildl., 4: 104-118.

18. Ganguly, A., Bhanot, V., Bisla, RS., Ganguly, I., Singh, H. and Chaudhri, S.S. (2015) Haematobiochemical alterations and direct blood polymerase chain reaction detection of Theileria annulata in naturally infected crossbred cows. Vet. World, 8(1): 24-28.

19. Hammer, J.F., Jenkins, C., Bogema, D. and Emery, D. (2016) Mechanical transfer of Theileria orientalis: Possible roles of biting arthropods, colostrum and husbandry practices in disease transmission. Parasit. Vectors, 9: 34.

20. Khatoon, S., Kolte, SW., Kurkure, NV., Chopde, NA. and Jahan, A. (2015) Detection of tropical bovine theileriosis by polymerase chain reaction in cattle. J. Parasit. Dis., 39(1): 53-56.

21. d'Oliveira, C., Van-der Weide, M., Habela, A., Jacquiet, P. and Jongejan, F. (1995) Detection of Theileria annulata in blood samples of carrier cattle by PCR. J. Clin. Microbiol., 33(10): 2665-2669.

22 Kamau, J., de Vos, A.J., Playford, M., Salim, B., Kinyanjui, P. and Sugimoto, C. (2011) Emergence of new types of Theileria orientalis in Australian cattle and possible cause of theileriosis outbreaks. Parasit. Vectors., 4: 22. 